\title{
Fluid Flow-Induced Calcium Response in Early or Late Differentiated Osteoclasts
}

\author{
Ping Li, ${ }^{1}$ Man Hu, ${ }^{2}$ Shujin Sun, ${ }^{2}$ Yan Zhang, ${ }^{2}$ Yuxin Gao, ${ }^{2}$ Mian Long, ${ }^{2}$ Bo Huo, ${ }^{2}$ and Ding Zhang ${ }^{1}$ \\ ${ }^{1}$ Department of Stomatology, Faculty of Surgery, Peking Union Medical College Hospital, Chinese Academy of Medical \\ Sciences, Beijing 100730, People's Republic of China; and ${ }^{2}$ Key Laboratory of Microgravity, Center for Biomechanics and \\ Bioengineering, Institute of Mechanics, Chinese Academy of Sciences, No. 15 North 4th Ring Road, Beijing 100190, \\ People's Republic of China
}

(Received 13 November 2011; accepted 17 March 2012; published online 25 April 2012)

Associate Editor Cheng Dong oversaw the review of this article.

\begin{abstract}
Intracellular calcium oscillation caused by receptor activator of nuclear factor kappa-B ligand has been demonstrated to promote the differentiation of osteoclasts. Osteoclasts are recruited on the surface of trabeculae, and are exposed to fluid flow caused by the deformation of the bone matrix. However, the roles of fluid shear stress (FSS) on calcium response during the differentiation process of osteoclasts are still unknown. In the current study, the formation of tartrate-resistant acid phosphatase-positive, multinucleated osteoclasts from RAW264.7 macrophage cells were induced by co-culturing them with the conditioned medium from MC3T3-E1 osteoblasts. The in situ observations showed a high correlation between the area and the nuclear number of osteoclasts. The cells were stimulated by FSS at different levels ( 1 or 10 dyne $\left./ \mathrm{cm}^{2}\right)$ before ( 0 day) or after being induced for 4 or 8 days. The mechanically-induced calcium response was recorded and analyzed. The results indicated a different property of calcium oscillation for the osteoclasts in different fusion stages (i.e., more calciumresponsive peaks appeared in small osteoclasts than those in the larger ones). The rates of calcium influx decreased and the time of recovery in osteoclast cytosol increased along with the fusion of osteoclasts. In addition, increasing the FSS level enhanced the calcium oscillation of osteoclasts at early induction (4 days). However, this effect was weakened at the late induction ( 8 days). The present work could help provide understanding regarding the mechanism of the involvement of calcium in mechanically induced bone remodeling.
\end{abstract}

Keywords-Osteoclasts, Fluid shear stress, Calcium oscillation, Induction time, Differentiation.

Address correspondence to Bo Huo, Key Laboratory of Microgravity, Center for Biomechanics and Bioengineering, Institute of Mechanics, Chinese Academy of Sciences, No. 15 North 4th Ring Road, Beijing 100190, People's Republic of China and Ding Zhang, Department of Stomatology, Faculty of Surgery, Peking Union Medical College Hospital, Chinese Academy of Medical Sciences, Beijing 100730, People's Republic of China. Electronic mail: huobo@ imech.ac.cn, dingz@china.com.cn

\section{INTRODUCTION}

Calcium signaling in osteoclasts regulates numerous cellular functions, such as differentiation, proliferation, migration, and bone resorption. The binding of the receptor activator of nuclear factor kappa-B ligand (RANKL) with the membrane receptor activator of nuclear factor kappa-B on an osteoclast could induce intracellular calcium oscillations, ${ }^{9}$ which would last until the formation of multinucleated osteoclasts. ${ }^{13}$ Calcium oscillation is closely related to the differentiation stages of osteoclasts, supported by the fact that the ability of calcium oscillation of osteoclasts at early differentiation is stronger than that at late differentiation. ${ }^{15}$ The elevation of intracellular calcium concentration activates calcineurin, which further leads to the activation of the nuclear factor of activated T-cells, cytoplasmic 1 (NFATc1). NFATc1 can upregulate the expression of tartrate-resistant acid phosphatase (TRAP), cathepsin $\mathrm{K}$, and $\beta 3$ integrin; thus, osteoclastogenesis and bone absorption are enhanced. ${ }^{13}$ The above results indicated that calcium oscillation is an important index of osteoclastic activity and is closely correlated with the differentiation stages.

The bone contains a large number of cavities, such as lacunar space and canaliculi, which are fully filled with tissue liquid. When the bone tissue is loaded, the deformation of the bone matrix drives the fluid to flow on the surface of the trabeculae or lacunae, and bone cells residing on these surfaces experience fluid shear stress (FSS). Some in vivo ${ }^{5,6}$ and in vitro ${ }^{11}$ studies revealed that FSS could activate the differentiation of osteoclasts and bone resorption. A few studies showed that some types of mechanical stimulations, such as the single-cell indentation by a glass pipette ${ }^{18-20}$ or whole-cell membrane stretching by changing the 
osmotic pressure, ${ }^{17}$ could lead to significant intracellular calcium response in osteoclasts. The increase of cytosolic calcium concentration responding to mechanical stimulations are mainly from the influx of extracellular calcium and the release of intracellular calcium stores, such as endoplasmic reticulum (ER) in osteoclasts. ${ }^{19}$ The intercellular calcium signaling between osteoblasts and osteoclasts can be found when both types of cells are cocultured. ${ }^{7}$ However, no studies concerning the relationship between FSS and calcium response in osteoclasts are available.

Osteoclasts originate from hematopoietic stem cells characterized by fusing monocytes into multinucleated giant cells. The presence of osteoblasts is necessary for this differentiation process, ${ }^{9}$ which is mainly due to the regulating roles of the secretions of osteoblasts containing macrophage colony-stimulating factor (M-CSF) and RANKL. Either the nuclear number of osteoclasts or the induction time is used to judge the stage of osteoclastic differentiation. However, the cellular behavior at various differentiation stages lacks adequate investigations. In addition, the calcium response under mechanical stimulations at different induction times and fusion stages is still unknown.

In the present work, the differentiation of RAW264.7 macrophage cells to osteoclasts was induced and the calcium response of osteoclasts with different nuclear numbers $(1,2-5$, and $\geq 6)$ at different induction times $(0,4$, and 8 days) under different levels of FSS ( 1 and 10 dyne $/ \mathrm{cm}^{2}$ ) was observed. The current experimental results showed that the FSS-induced calcium oscillation occurred in small osteoclasts but disappeared in the large ones, suggesting a correlation between the oscillation property of osteoclasts and their differentiation stages.

\section{MATERIALS AND METHODS}

\section{Materials}

The MC3T3-E1 cells were purchased from the American Type Culture Collection (ATCC, Manassas, USA). The RAW264.7 cells were purchased from the European Collection of Cell Cultures (ECACC, Wiltshire, UK). Dulbecco's Modified Eagle's Medium (DMEM/high glucose), alpha modified Minimum Essential Medium ( $\alpha$-MEM), phosphate buffered saline (PBS), trypsin-EDTA, penicillin/streptomycin, and L-glutamine were provided by Hyclone (Thermo Scientific, Beijing, China). The Mouse TRANCE/RANKL/ TNFSF11 Quantikine ELISA kit and Mouse M-CSF Quantikine ELISA kit were provided by R\&D System (Minneapolis, USA). Dimethyl sulfioxide (DMSO) and TRAP Staining Kit were purchased from Sigma
(St. Louis, USA). Thermanox plastic coverslips were purchased from NUNC (Roskilde, Denmark). Fetal bovine serum (FBS), Fluo-4 AM, Pluronic F-127, and Hoechst 33342 were purchased from Invitrogen (Eugene, USA).

\section{Conditioned Medium from Osteoblasts}

The MC3T3-E1 cells were seeded in dishes at a density of $2 \times 10^{4}$ cells $/ \mathrm{cm}^{2}$ and maintained in $\alpha$-MEM supplemented with $10 \%$ heat-inactivated FBS and $1 \%$ penicillin-streptomycin solution at $37^{\circ} \mathrm{C}$ in a humidified atmosphere of $5 \% \mathrm{CO}_{2}$. The medium was changed every $48 \mathrm{~h}$, and the conditioned medium was collected. The RANKL content in the conditioned medium was measured using Quantikine Mouse RANKL immunoassay. The $50 \mu \mathrm{L}$ conditioned medium sample with $50 \mu \mathrm{L}$ assay diluent in the microplate coated with RANKL antibody was incubated for $2 \mathrm{~h}$ at room temperature. After aspirating and washing each well five times, $100 \mu \mathrm{L}$ substrate solution mixed with color reagents $\mathrm{A}$ and $\mathrm{B}$ was added to each well and incubated for $30 \mathrm{~min}$. The optical density at $450 \mathrm{~nm}$ was obtained and the concentration of RANKL from a standard curve was calculated. The procedure was repeated three times.

\section{Induction and Identification of Osteoclast Differentiation}

RAW264.7 cells were seeded at a density of $2 \times 10^{4}$ cells $/ \mathrm{cm}^{2}$ on plastic coverslips. After $24 \mathrm{~h}$, the medium was replaced with a $1: 1(\mathrm{v} / \mathrm{v})$ mixture of the conditioned medium and the growth medium of DMEM supplemented with $10 \%$ heat-inactivated FBS, $3.5 \mathrm{mM}$ L-glutamine, and $1 \%$ penicillin-streptomycin solution. The time point prior to the induction was described as ' 0 day'. The induction medium containing the conditioned medium was changed every other day. The multinucleated osteoclasts with more than 2 nuclei were visible after 2-days induction.

TRAP Staining Kit was used to identify the formation of TRAP-positive, multinucleated osteoclasts. A coverslip with cells was rinsed three times with PBS and then fixed with citrate-acetone solution for $30 \mathrm{~s}$ at room temperature. Next, the coverslip with the reaction solutions containing fast garnet GBC solution, naphthol AS-BI phosphate solution, acetate solution, and tartrate solution was incubated for $1 \mathrm{~h}$ at $37{ }^{\circ} \mathrm{C}$. The cells were then counterstained for $2 \mathrm{~min}$ with hematoxylin solution. Finally, the coverslip was allowed to dry in air, and the cells were observed under a microscope. The osteoclasts with active acid phosphatase showed purplish to dark red granules in the cytoplasm. 


\section{Cellular Spreading Area and Nuclear Number}

Hoechst dye was used to stain the cellular nuclei. The cells were first fixed with 3.7\% formaldehyde for $5 \mathrm{~min}$ and were stained with $0.2 \mathrm{mM}$ Hoechst for $10 \mathrm{~min}$ at room temperature after rinsing three times with PBS. The nuclear number of an osteoclast was counted in the fluorescent image and the spreading area of the same cell was measured through in situ light microscopic image. The calibrated correlation between the cellular spreading area and nuclear number can be used to estimate the nuclear number of multinucleated osteoclasts through their areas in calcium staining images, in which the nuclei of cells are invisible.

\section{Intracellular Calcium Response Under FSS}

After cells were seeded on a plastic coverslip at a density of $4 \times 10^{5}$ cells $/ \mathrm{cm}^{2}$ for 1 day the cytosolic calcium was stained with $5 \mu \mathrm{M}$ of Fuo-4 AM in DMSO and $0.02 \%$ Pluronic F-127 for $2 \mathrm{~h}$ in a culture medium at room temperature. The coverslip was rinsed with dye-free medium twice, and was mounted on a custom-made parallel plate flow chamber. The entire operation was performed with extreme caution to minimize the response of cells to early agitations. The subsequent 20-min resting period was shown to be sufficient for cells to recover the stable state for repetitive calcium responses. The calcium response of cells under the steady fluid flow stimulation was recorded with a charge-coupled device camera (IMAGO-QE) for 10-min (i.e., $1 \mathrm{~min}$ for baseline followed by the 9-min fluid flow stimulation). The fluorescence intensity of the calcium concentration for each cell was later normalized by its corresponding baseline.

\section{Statistical Analysis}

More than 10 coverslips were tested for a specific induction time and FSS level. In one group, 115-322 monocytes, $116-261$ osteoclasts (2-5 nuclei), and 9-18 osteoclasts ( $\geq 6$ nuclei) were analyzed. All the results were expressed as mean \pm standard error of the mean (SEM). Statistical differences between the mean values of different groups were determined using one-way ANOVA, and was considered significant when $p<$ 0.05 . The statistical analysis was performed using the ImageJ 1.44 and Origin 8.1 software.

\section{RESULTS}

The Conditioned Medium from MC3T3-E1 Cells is Capable of Inducing Formation of Osteoclasts

The ELISA assay showed that the concentration of soluble RANKL secreted from MC3T3-E1 cells cultured for $48 \mathrm{~h}$ in the conditioned medium was $32 \pm 4 \mathrm{pg} / \mathrm{mL}$. Almost all cells were TRAP-positive after the RAW264.7 macrophage cells were cultured in $50 \%$ conditioned medium from MC3T3-E1 osteoblasts (Fig. 1a). The Hoechst staining images merged with in situ light microscopic images revealed that multinucleated osteoclasts were formed (Fig. 1b). Less than 1\% multinucleated cells appeared in the non-induced RAW264.7 cells. However, the percentage of multinucleated cells increased to 7 or $9 \%$ after the 4 - or 8 -days induction, respectively, showing that increasing induction time enhanced the fusion of osteoclasts (Fig. 1c). The number density of cells for the 4- and 8-days induction decreased by 11 and $27 \%$ compared with that before the induction. However, no significant difference was found (Fig. 1d).

\section{The Size of Osteoclast is Correlated to Nuclear Number}

The number of multinucleate RAW264.7 cells prior to induction (Fig. 1c) was not calculated when the correlation between cellular size and nuclear number was analyzed because of rarity. Figure le shows that the average area of cells with same nuclear numbers was obviously independent of induction times. In the early fusion of osteoclasts, the cellular area increased from $120 \mu \mathrm{m}^{2}$ for monocytes to $710-760 \mu \mathrm{m}^{2}$ for 6 -nuclei osteoclasts. However, the increasing trend was not obvious in the late fusion of $\geq 6$ nuclei, during which the area of cells varied in the range of $700-900 \mu \mathrm{m}^{2}$. The cells were divided into three groups, i.e., monocytes, small (2-5 nuclei), and large ( $\geq 6$ nuclei) osteoclasts according to the area-nuclear number correlation. The box chart showed that the distribution of cellular area in the three groups was not symmetrically Gaussian (Fig. 1f). The dispersion of the cell size in 4-days induction group was larger than that in the 8-days induction group (Fig. 1f), indicating that more cells have undergone fusion in a shorter induction time. The dashed lines in Fig. If indicated the area thresholds to distinguish nuclear number, i.e., the cells with an area of less than $161 \mu \mathrm{m}^{2}$ were regarded as monocytes, with an area larger than $435 \mu \mathrm{m}^{2}$ as osteoclasts with $\geq 6$ nuclei, and the others as osteoclasts with 2-5 nuclei. Although the area distribution of these groups overlapped each other, the error should be lower than $10 \%$ (Fig. 1f). Fluo-4 AM staining did not change the cellular spreading area compared with the nucleus staining by Hoechst (data not shown). Thus, the nuclear number of cells can be estimated through the spreading area measured in calcium staining images.

\section{FSS Induces Calcium Response in RAW264.7 Cells and Osteoclasts}

The time-lapse intracellular calcium images showed that after 4-d induction, most of the cells produced 

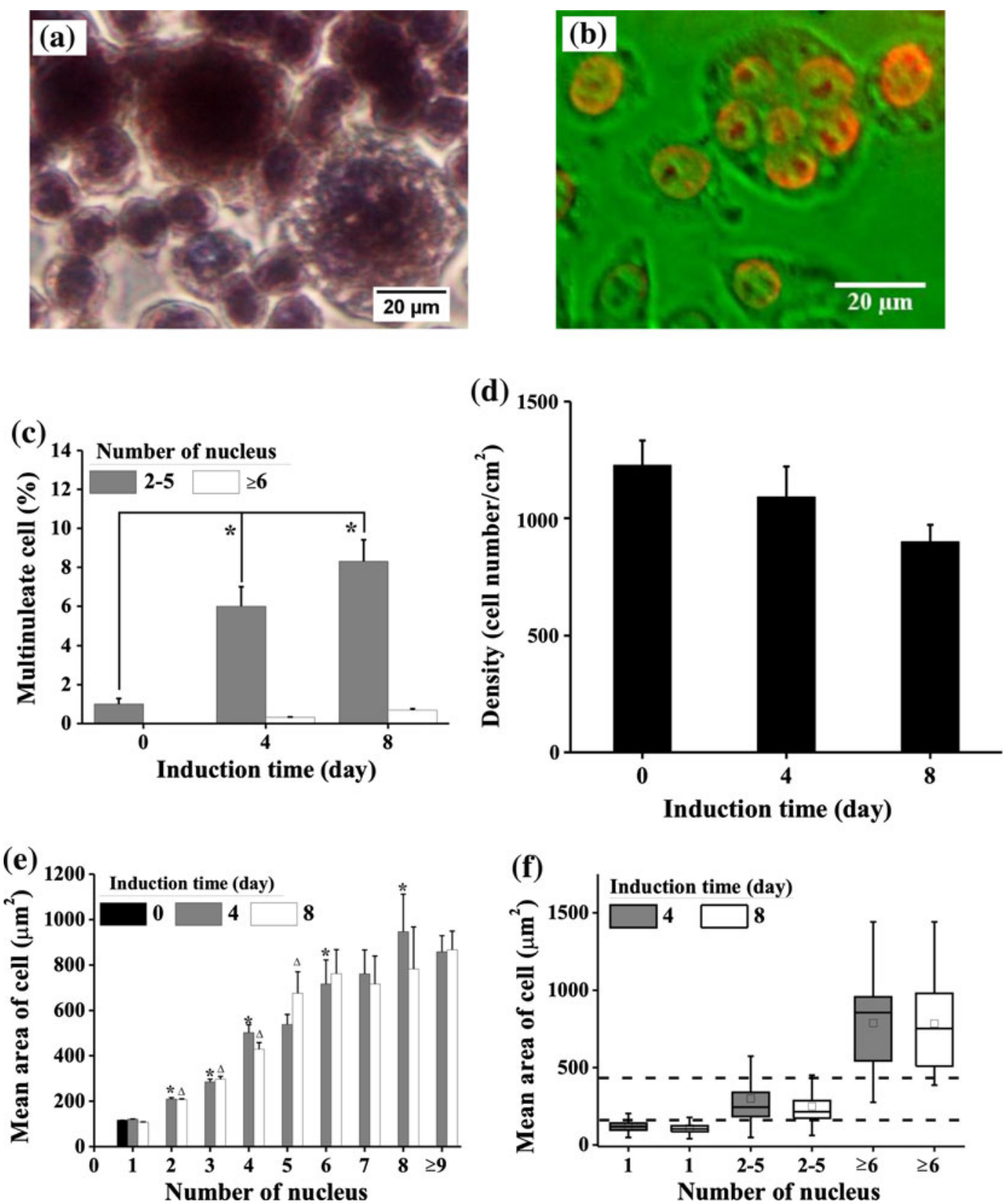

FIGURE 1. Identification of osteoclast formation and the correlation between cellular spreading area and nuclear number. (a) TRAP-positive cells after 4-days culture with conditioned medium from MC3T3-E1; (b) the merged Hoechst staining (red) and light microscopic (green) images after the 4-days induction; (c) the percentage of multinucleated cells (2-5 nuclei and $\geq 6$ nuclei) at different times of 0,4 , and 8 days $\left({ }^{*} p<0.05\right.$ ); (d) the number density of cells at different times of 0,4 , and 8 days; (e) the correlation between spreading area and nuclear number at different times of 0,4 , and 8 days ( ${ }^{*}$ and $\Delta, p<0.05, n$-nuclei group compared with $(n-1)$-nuclei group at 4- and 8-days induction, respectively; (f) box chart showing the distribution of the spreading area of monocytes, small (2-5 nuclei), and large osteoclasts ( $\geq 6$ nuclei), in which the ends of whiskers represent the 5th and 95th percentiles and the square denotes the mean value. The horizontal dashed lines in (f) distinguish the area ranges used to identify the nuclear number.

calcium response to FSS $\left(1 \mathrm{dyne} / \mathrm{cm}^{2}\right)$, and some cells presented multiple responses (Fig. 2a). Figure $2 b$ showed the typical calcium response curves of osteoclasts with different nuclear numbers to two FSS levels and varying induction times. After the onset of fluid flow, the cytosolic calcium concentration in most cells increased quickly to a maximum and then decreased to the baseline, producing a responsive peak. Some cells have multiple responsive peaks called calcium oscillation. Figure $2 \mathrm{~b}$ revealed that the profiles of calcium response curves were influenced by shear levels, induction times, and nuclear numbers. To further 
(a)
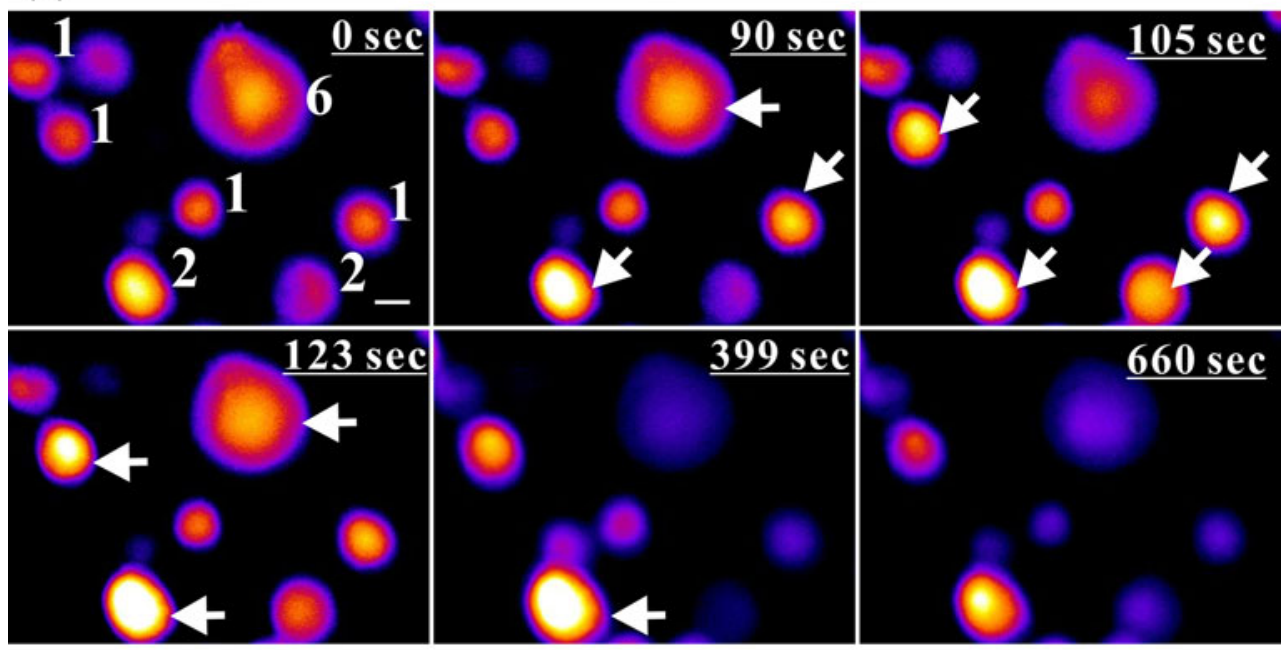

(b)

$1 \mathrm{dyne} / \mathrm{cm}^{2}$

10 dyne $/ \mathrm{cm}^{2}$
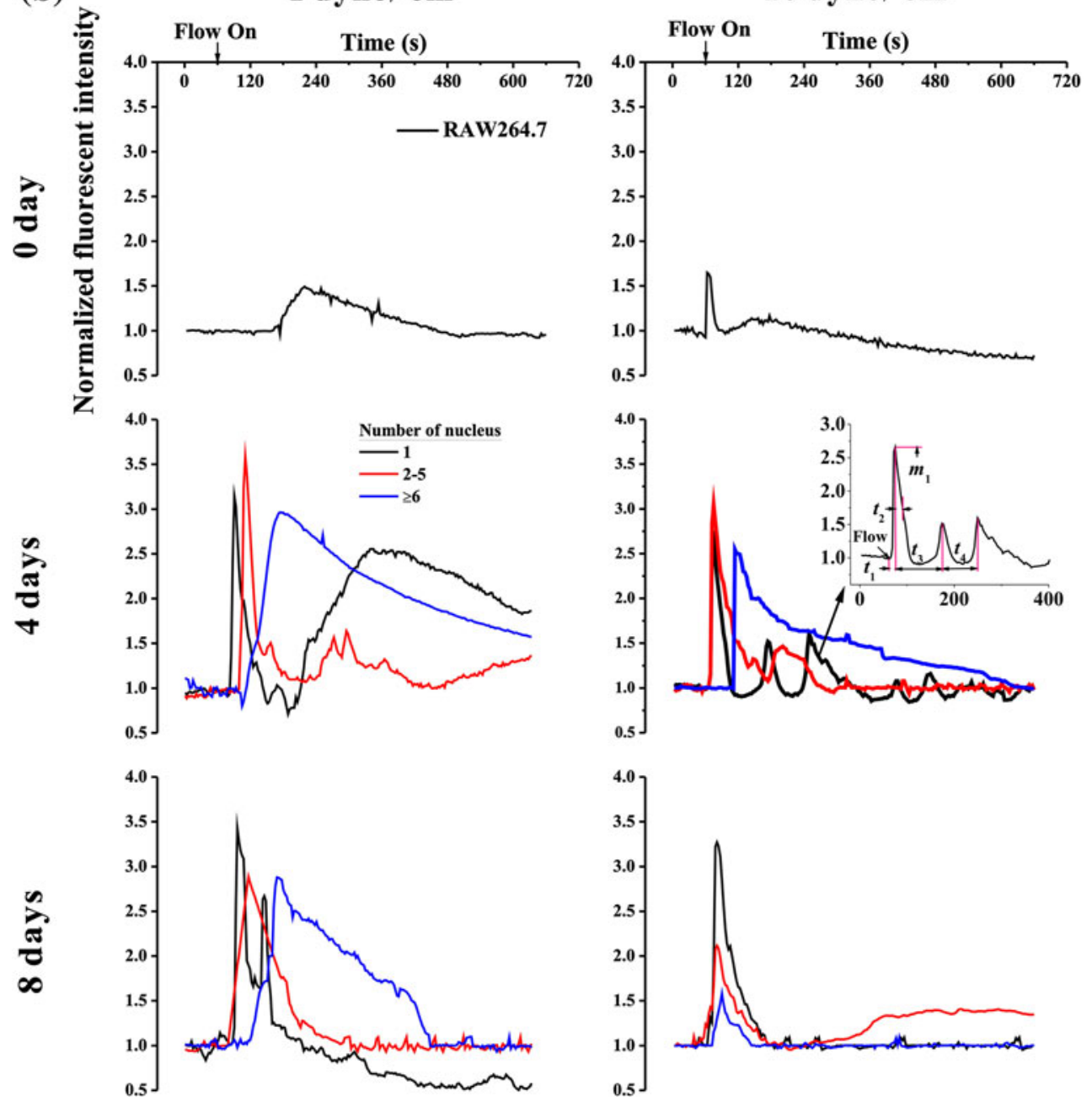

FIGURE 2. (a) Pseudo-color images of intracellular calcium staining for monocytes and multinucleated osteoclasts under FSS of $1 \mathrm{dyne} / \mathrm{cm}^{2}$ after 4-days induction. The digits beside the cells indicate the number of nuclei. The arrowheads point to those responding cells. Some cells were able to respond multiple times (scale bar: $10 \mu \mathrm{m}$ ). (b) Typical curves of calcium response in cells with different nuclei under different FSS levels and induction times. The inset shows the definition of characteristic parameters. 
quantitatively analyze the characteristics of calcium response, some parameters (see the inset of Fig. 2b) were defined. The number of responsive peaks indicates the calcium oscillation capability of a cell. The time to the first peak $(t 1)$ denotes the speed of calcium response to FSS. The magnitude of the first peak $(\mathrm{m} 1)$ of normalized fluorescent intensity reveals the relative amount of calcium influx into cytosol. The time from the first peak to its $50 \%$ relaxation point $(t 2)$ shows the speed of calcium recovery to the base level. The time intervals between peaks $(t 3, t 4)$ reflect the speed of calcium oscillation.

\section{FSS-Induced Calcium Oscillation Reduces in Late Differentiated Osteoclasts}

Figures $3 \mathrm{a}$ to $3 \mathrm{c}$ showed that the calcium responsiveness of cells depends on induction time, FSS level, and nuclear number. Prior to induction, 37 and $23 \%$ of monocytes could not respond to 1 and $10 \mathrm{dyne} / \mathrm{cm}^{2}$ FSS, respectively (Fig. 3a). After being induced with the conditioned medium, the proportion of nonresponsive cells significantly decreased to less than $10 \%$ for monocytes or $2-5$ nuclei cells, and this decreasing trend was enhanced by the increasing FSS level (Figs. 3b and 3c). However, in the early
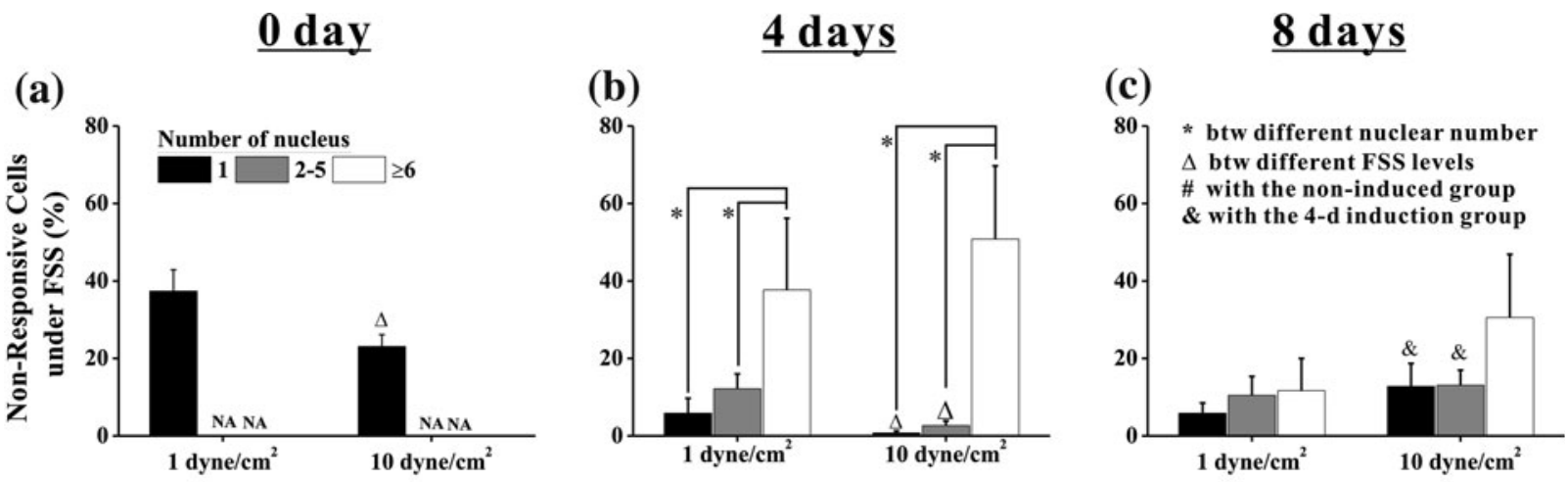

(d)

(e)
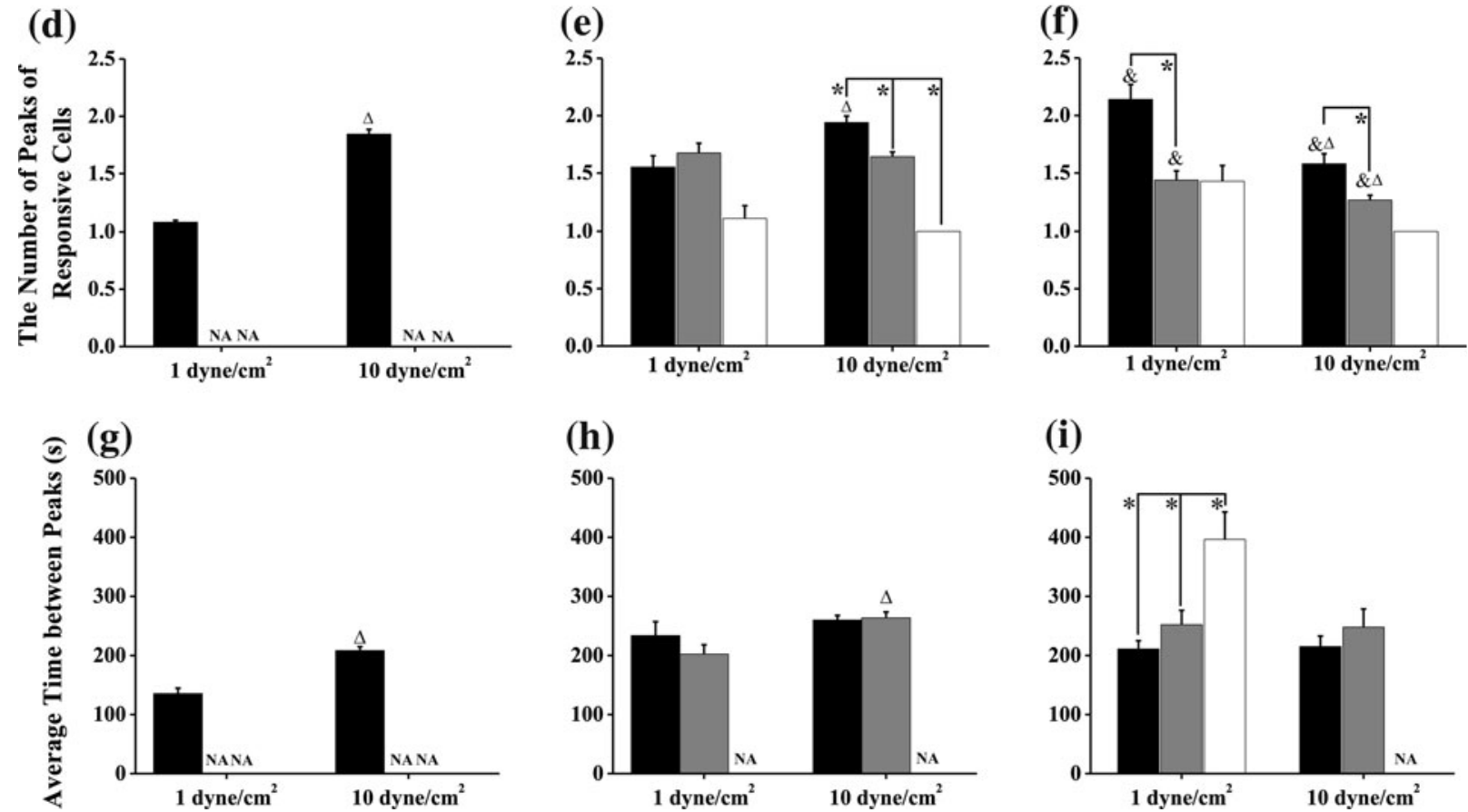

FIGURE 3. Proportion of non-responsive cells under FSS stimulations ((a)-(c)) and the properties of calcium oscillation in cells; (d)-(f) shows the number of calcium responsive peaks, and $(g)-(i)$ shows the average time between multiple peaks. The following symbols represent significant difference $(p<0.05)$ when changing only one factor and fixing the other two factors: * among groups with different nuclear number; $\Delta$ among groups under different FSS levels; " with the non-induced group; and \& with the 4-days induction group. 
induction, the larger multinucleate cells with $\geq 6$ nuclei had weaker responsiveness, and about $38-50 \%$ of the cells showed no response to the FSS stimulation.

At low FSS level of 1 dyne $/ \mathrm{cm}^{2}$, there were $62 \%$ non-induced RAW264.7 cells producing at least one calcium response peak (Fig. 3d). After 4- or 8-days induction, the average number of responsive peaks for monocytes increased to 1.6 or 2.1 (Figs. $3 e$ and 3f), respectively, which were significantly higher than that of the non-induced cells $(p<0.05)$. However, the calcium oscillation abilities of osteoclasts depended on the FSS levels. In non-induced and 4-days induction groups, higher FSS level increased the number of peaks. By contrast, this number decreased in the 8 -days induction group to $1.6,1.3$, and 1 for monocytes, osteoclasts with $2-5$, and $\geq 6$ nuclei, respectively. The result showed that the calcium oscillation property of osteoclasts at different induction time differs with FSS levels.

In the 4- or 8-days induction groups, the number of calcium responsive peaks significantly decreased when the cells fused into larger osteoclasts (Figs. 3e and 3f). The number of peaks in large osteoclasts with $\geq 6$ nuclei reduced to 1.1 for the 4-days group and 1.4 for the 8-days group under low FSS. Under high FSS, the large osteoclasts only had one calcium peak. These results showed that the calcium oscillation ability of the cells was significantly reduced in the late fusion of osteoclasts.

The time intervals between the responsive peaks became longer for the non-induced RAW264.7 cells with increasing FSS level (Fig. 3g), indicating that cells need more time to recruit the cytosolic calcium ions back into the intracellular calcium stores or to pump them out of cells, preparing for a subsequent peak. The nuclear number of cells had no influence on the time intervals except that the larger osteoclasts took longer time to have multiple responses under low FSS level at the late induction time (8 days).

\section{The Sensitivity of Calcium Response to FSS Reduces in Late-Differentiated Osteoclasts}

In the non-induced group under $1 \mathrm{dyne} / \mathrm{cm}^{2} \mathrm{FSS}$, RAW264.7 cells needed about $118 \mathrm{~s}$ to reach their first responsive peak with the magnitude of 2 (Figs. 4a and $4 \mathrm{~g})$, then took $113 \mathrm{~s}$ to go down from the peak to the $50 \%$ relaxation point (Fig. $4 \mathrm{~d}$ ). When FSS increased to 10 dyne $/ \mathrm{cm}^{2}$, RAW264.7 cells required a much shorter time of $23 \mathrm{~s}$ to the first peak and quickly relaxed to $50 \%$ in $30 \mathrm{~s}$.

Under low FSS level, the induction by the conditioned medium enhanced both the speeds of calcium influx and recovery in monocytes. In the 4-days induction group, monocytes took $47 \mathrm{~s}$ to produce the first peak (Fig. 4b) and $44 \mathrm{~s}$ to relax to $50 \%$ (Fig. 4e), and both times were shorter by $60 \%$ compared with the non-induced group, whereas the magnitude of the first peak of normalized fluorescent intensity was higher by $30 \%$ (Fig. 4h). When FSS level increased, calcium influx or recovery time in the induced monocytes had no obvious changes except for the magnitude of the first peak, which increased to $30 \%$ compared with non-induced cells. In addition, the induction time had no influence on the corresponding parameters of the first peak (Figs. 4c, 4f, and 4i). These results showed that the conditioned medium from osteoblast could improve the magnitude of calcium responsive peaks and accelerate the speed of calcium influx and recovery under low FSS, but not under high FSS.

The ability of calcium influx and recovery of osteoclasts was significantly reduced along with their fusion into larger cells. In the 4-days induction group under low FSS, small and large osteoclasts took longer time to get to their first peaks by 51 and $83 \%$ compared with monocytes, respectively (Fig. $4 \mathrm{~b}$ ), as well as longer time to the reach $50 \%$ relaxation point by 45 and $163 \%$, respectively. With increased FSS level, the response and relaxation times between monocytes and small osteoclasts had no significant difference, but became longer in osteoclasts with $\geq 6$ nuclei (Figs. $4 b$, $4 \mathrm{c}, 4 \mathrm{e}$, and $4 \mathrm{f}$ ). In the 8-days induction group under low FSS, the slowing-down trend of calcium response and recovery was similar to the early induction. However, under high FSS, there was no significant difference among osteoclasts in different fusion stages. After the addition of the induction medium, the magnitudes of the first peaks showed no relationship with fusion stages and induction times. These results indicated that osteoclasts in different fusion stages had varying ability of responding to FSS.

\section{DISCUSSION}

In the current study the conditioned medium from osteoblasts was adopted to stimulate the differentiation of osteoclasts. The previous studies have demonstrated that hematopoietic stem cells could differentiate into multinucleated, bone-resorbing osteoclasts induced by both M-CSF and RANKL. ${ }^{2,10}$ However, in some cases, e.g., when knocking down IP3R2/3 channel proteins, bone marrow macrophage cells were not capable of differentiating into osteoclasts even in the presence of RANKL and M-CSF. The experimental results also showed that the differentiation process restarted when the cells were cocultured with osteoblasts, ${ }^{9}$ which means that some factors excluding RANKL and M-CSF from osteoblasts or direct contact with osteoblasts may be critical in the regulation 
(a)

\section{o day}
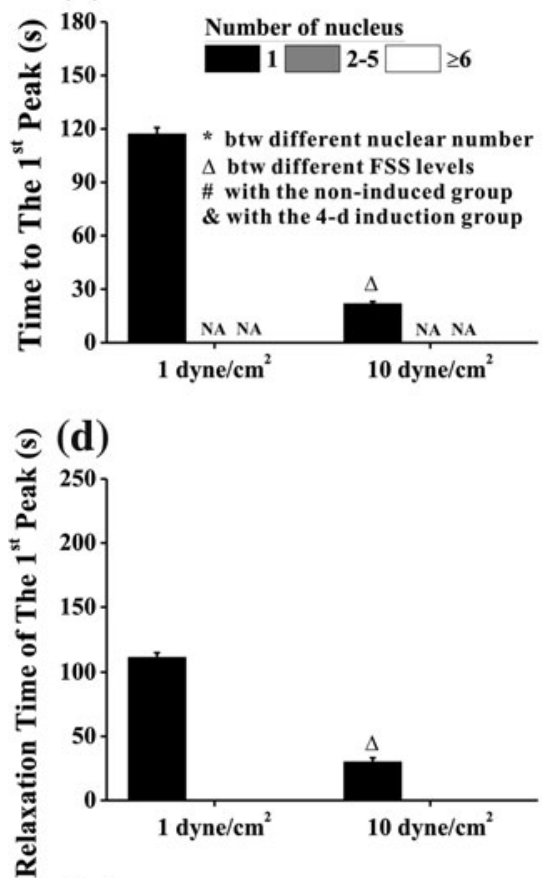

(g)

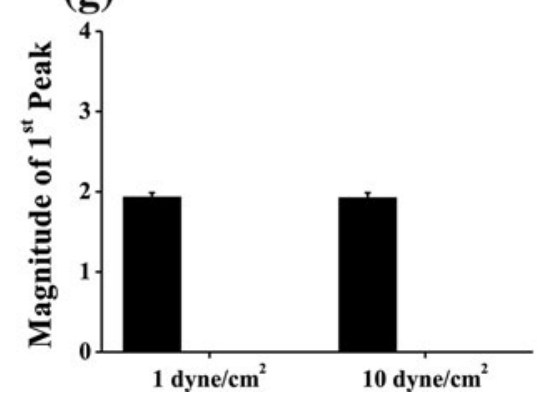

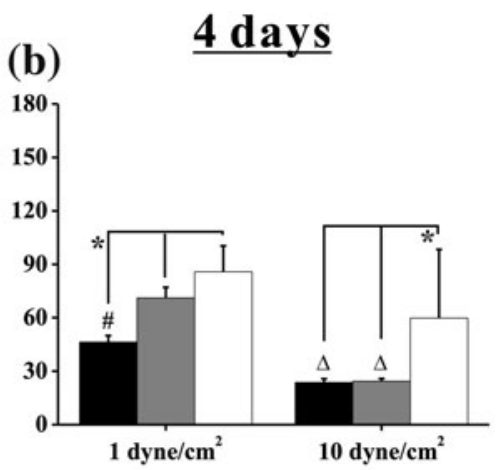

(e)

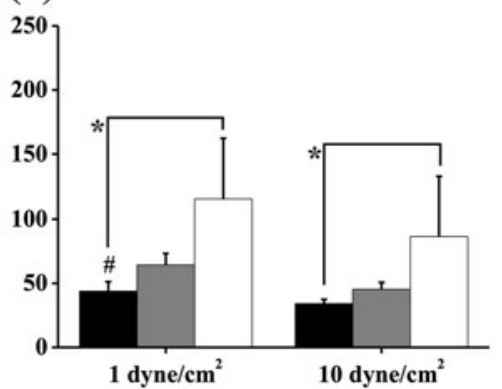

(h)

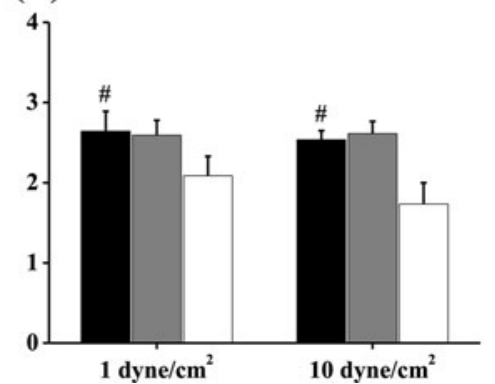

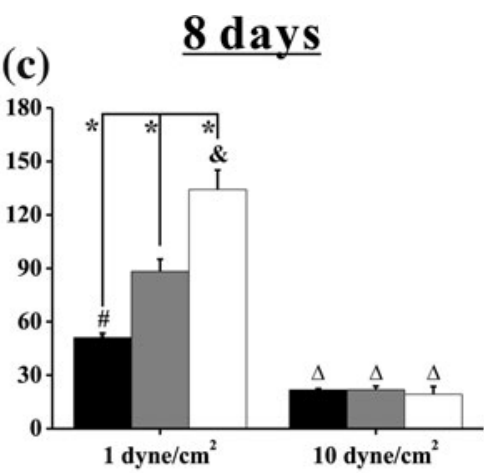

(f)

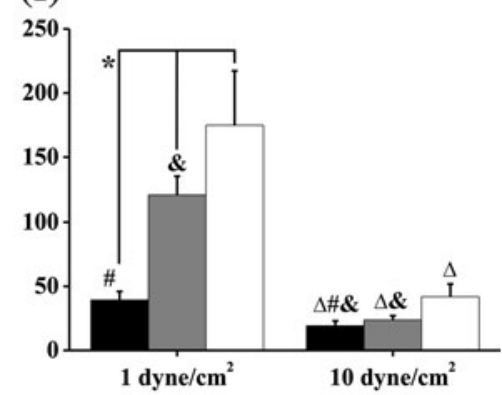

(i)

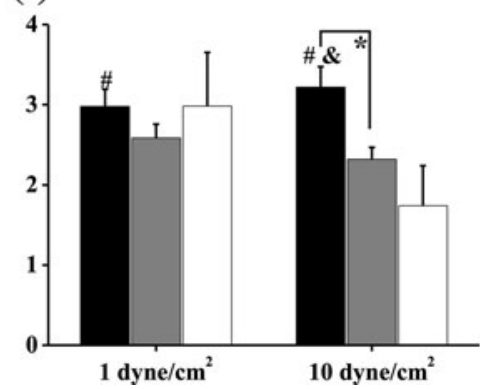

FIGURE 4. The properties of the first calcium responsive peaks: (a)-(c) shows the time to reach the first peak, (d)-(f) shows the time to the $50 \%$ relaxation point of first peak, and (g)-(i) shows the magnitude of the first peak of normalized fluorescent intensity. The following symbols represent significant difference $(p<0.05)$ when changing only one factor and fixing the other two factors: * among groups with different nuclear number; $\Delta$ among groups under different FSS levels; \# with the non-induced group; and $\&$ with the 4 days-induction group.

of osteoclast differentiation. Therefore, culturing osteoclast precursors with osteoblast secretion may be an approach close to an in vivo condition for their differentiation. The TRAP results of the current study demonstrated that the conditioned medium successfully induced the differentiation of RAW264.7 macrophage cells into TRAP-positive, multinucleated osteoclasts after $48 \mathrm{~h}$ induction. This regulation role of the conditioned medium may be due to RANKL. The amount of RANKL in the conditioned medium $(32 \pm 4 \mathrm{pg} / \mathrm{mL})$ was measured, which was similar to previous measurement results of $10 \mathrm{pg} / \mathrm{mL}$ RANKL secreted by MC3T3-E1 cells cultured for $24 \mathrm{~h} .{ }^{16}$ When we incubated RAW264.7 cells with $20 \mathrm{ng} / \mathrm{mL}$ RANKL, the number of RANKL-induced multinucleated osteoclasts didn't have significant difference with that of osteoblasts-induced formation of osteoclasts after 4-days induction but 8-days incubation with RANKL induced about $12 \%$ of multinucleate cell, which is significantly higher than $9 \%$ of multinucleate cell induced by conditioned medium from osteoblasts (data not shown).

Researchers usually use the indexes of induction time or nuclear number to identify the differentiation stages for monocytes/macrophage cells to multinucleated osteoclasts. One study found that RAW264.7 macrophage cells with soluble RANKL and primarily isolated hematopoietic stem cells with M-CSF and RANKL showed positive TRAP staining after 4-days culture, and can maintain the osteoclastic lineage for $10-16$ days. ${ }^{15}$ When RANKL was used during induction, the formation of osteoclasts with more than 3 
nuclei showed the first peak at 4 days of induction, but the number of osteoclasts in apoptosis increased and achieved the first apoptosis peak at 8-days induction, thus the density of osteoclasts decreased about sevenfold compared with the 4-days induction. ${ }^{1}$ Therefore, the 4-days induction made monocytes more active to differentiate into mature multinucleated osteoclasts than the induction for 8-10 days. The same reason was also used when the 4-days induction was chosen as the early stage of differentiation of osteoclasts and 8-days induction as the late stage. Being consistent with this observation, Hoechst staining results of the present study showed that the apoptotic fragments of cells after the 8-days induction were obviously more than those after the 4-days induction (data not shown). The number of nuclei is another index to identify the differentiation stage of an osteoclast. One study showed that osteoclasts with $\leq 4,5-10$, and $>10$ nuclei had different number of calcium oscillation caused by RANKL, concluding that the ability of calcium oscillation in the early differentiation was stronger than that of the late differentiation. ${ }^{12}$ The current experimental results demonstrated that calcium oscillation under FSS in large osteoclasts was notably weaker than those in monocytes and small osteoclasts. These data showed that calcium oscillations attenuated at late fusion under either RANKL- or FSS-stimulations. The results also revealed that the nuclear number of osteoclasts could be a better index of differentiation than induction time because the calcium response under FSS was consistent at different fusion stages. Given that longer induction caused higher ratio of multinucleated osteoclasts in all cells, induction time will be a complementary index in identifying the differentiation stages of osteoclasts.

There are still no reports about the calcium response of osteoclasts to FSS to date. Thus, the present study will provide fundamental data for deeply understanding the regulation mechanism of the mechanical stimulations on bone resorption of osteoclasts. The increase of FSS level at late fusion reduced the calcium oscillation. Given that calcium oscillation could enhance the resorption ability of osteoclasts, ${ }^{15}$ high FSS level might inhibit the functions of osteoclasts. Some studies showed that high mechanical stretching strain can inhibit the formation of osteoclasts, ${ }^{8,14}$ but a cell-level experiment demonstrated that TRAP activity and the number and size of resorption pits increased with increasing FSS $\left(6-20\right.$ dyne $\left./ \mathrm{cm}^{2}\right)$ on osteoclasts. ${ }^{11}$ Therefore, it is necessary to clarify the correlation among FSS levels, calcium response of osteoclasts, and bone resorption in the future.

Some studies also demonstrated that RANKL could lead to calcium oscillation in the cytosol of osteoclasts, and that the oscillation in small osteoclasts ( $<5$ nuclei) was stronger than larger osteoclasts. ${ }^{12}$ More than $60 \%$ cells with $<5$ nuclei had calcium oscillation, but this value decreased to $40 \%$ for osteoclasts with 5-10 nuclei.

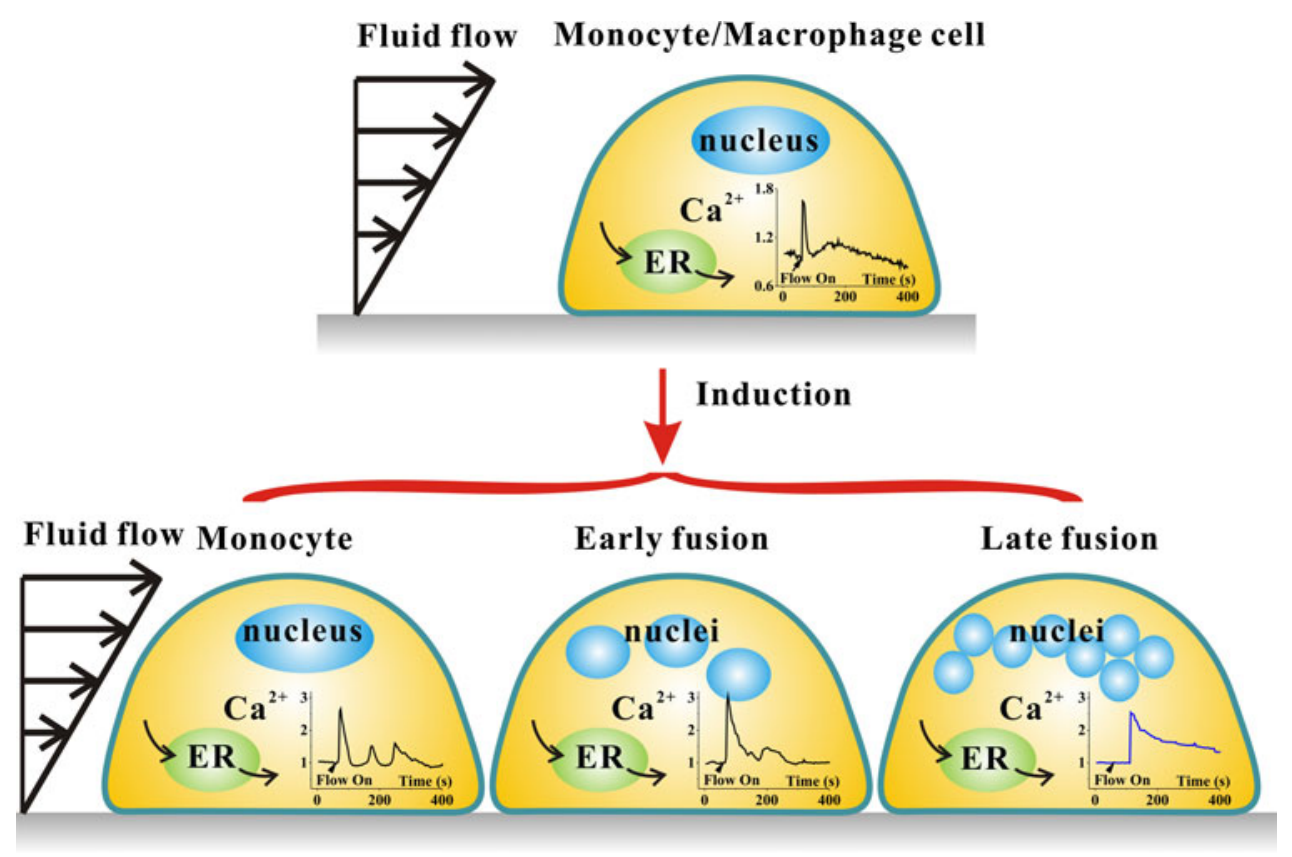

FIGURE 5. A schematic model showing the profiles of the calcium responses in osteoclasts exposed to FSS in different fusion stages. In non-induced stage, RAW264.7 monocytes/macrophage cells have single calcium responsive peak. In the early fusion after induction, monocytes and small osteoclasts show calcium oscillation, but in the late fusion large osteoclasts only responded once or did not have any response to the FSS stimulation. 
No oscillation was observed in osteoclasts with $>10$ nuclei. The frequency of calcium oscillation was statistically 0.8 peaks per minute and the amplitude was about $17 \%$ higher than the baseline. The form of stimulation (chemical or mechanical) and its magnitude decides the pattern of calcium oscillation in osteoclasts, which induces more downstream signaling molecules, such as NFATC1, necessary for osteoclastogenesis. ${ }^{3,4}$ The current results showed that FSS could induce calcium response in osteoclasts, which was obviously different with that induced by $100 \mathrm{ng} / \mathrm{mL}$ RANKL after 5 -days incubation shown in another study. ${ }^{12}$ The amplitude of calcium responsive peaks induced by FSS was five times of that induced by RANKL. However, the time interval between peaks for FSS was five times longer than RANKL. The calcium peaks induced by RANKL were lower and sharper, and the average time length of peaks was about 20 s. Furthermore, the time length of peaks induced by FSS varied from 30 to $120 \mathrm{~s}$. To prevent the coupled effect of RANKL and FSS from calcium response during the current experiment, the conditioned medium from osteoblasts that should contain RANKL when exerting FSS to cells was not used. Similar to the calcium response induced by RANKL,${ }^{12}$ the current results proposed that FSS can induce calcium oscillation in small osteoclasts with $<6$ nuclei, but only caused single calcium peak or even no calcium response in large osteoclasts (see Fig. 5). If the calcium oscillation caused by FSS could promote osteoclastogenesis and bone resorption, the above results will conclude that high FSS may activate the resorption ability of small osteoclasts and inhibit the function of larger osteoclasts.

In conclusion, the current results showed that under FSS stimulations, more calcium responsive peaks were found in small osteoclasts than in the larger osteoclasts. Increasing FSS enhanced the calcium oscillation of osteoclasts at early induction, but impeded it at late induction. Furthermore, the calcium responsive sensitivity of osteoclasts to FSS was reduced along with the fusion of osteoclasts.

\section{ACKNOWLEDGMENTS}

This work was supported by the National Natural Science Foundation of China [30970707 (BH) and 31070829 (DZ)], the National Key Basic Research Foundation of China (2011CB710904), and the Knowledge Innovation Project of the CAS (KJCX2YW-L08) (ML).

\section{CONFLICT OF INTEREST}

No conflict of interested is assigned to the manuscript.

\section{REFERENCES}

${ }^{1}$ Akchurin, T., T. Aissiou, et al. Complex dynamics of osteoclast formation and death in long-term cultures. PLoS ONE 3(5):11, 2008.

${ }^{2}$ Arai, F., T. Miyamoto, et al. Commitment and differentiation of osteoclast precursor cells by the sequential expression of c-Fms and receptor activator of nuclear factor kappaB (RANK) receptors. J. Exp. Med. 190(12): 1741-1754, 1999.

${ }^{3}$ Dolmetsch, R. E., R. S. Lewis, et al. Differential activation of transcription factors induced by $\mathrm{Ca} 2+$ response amplitude and duration. Nature 386(6627):855-858, 1997.

${ }^{4}$ Dolmetsch, R. E., K. Xu, et al. Calcium oscillations increase the efficiency and specificity of gene expression. Nature 392(6679):933-936, 1998.

${ }^{5}$ Fahlgren, A., M. P. G. Bostrom, et al. Fluid pressure and flow as a cause of bone resorption. Acta Orthop. 81(4): 508-516, 2010

${ }^{6}$ Johansson, L., U. Edlund, et al. Bone resorption induced by fluid flow. J. Biomech. Eng. Trans. ASME 131(9):5, 2009.

${ }^{7}$ Jorgensen, N. R. Short-range intercellular calcium signaling in bone. APMIS Suppl 118:5-36, 2005.

${ }^{8}$ Kurata, K., T. Uemura, et al. Mechanical strain effect on bone-resorbing activity and messenger RNA expressions of marker enzymes in isolated osteoclast culture. J. Bone Miner. Res. 16(4):722-730, 2001.

${ }^{9}$ Kuroda, Y., C. Hisatsune, et al. Osteoblasts induce Ca2+ oscillation-independent NFATc1 activation during osteoclastogenesis. Proc. Natl. Acad. Sci. USA 105(25):86438648, 2008

${ }^{10}$ Lacey, D. L., E. Timms, et al. Osteoprotegerin ligand is a cytokine that regulates osteoclast differentiation and activation. Cell 93(2):165-176, 1998.

${ }^{11}$ Liu, Y., L. Li, et al. Effects of fluid shear stress on bone resorption in rat osteoclasts. J. Biomed. Eng. 24(3):544-548, 2007.

${ }^{12}$ Masuyama, R., J. Vriens, et al. TRPV4-mediated calcium influx regulates terminal differentiation of osteoclasts. Cell Metab. 8(3):257-265, 2008.

${ }^{13}$ Negishi-Koga, T., and H. Takayanagi. Ca2+-NFATc1 signaling is an essential axis of osteoclast differentiation. Immunol. Rev. 231:241-256, 2009.

${ }^{14}$ Rubin, J., X. Fan, et al. Osteoclastogenesis is repressed by mechanical strain in an in vitro model. J. Orthop. Res. 17(5):639-645, 1999.

${ }^{15}$ Sakai, H., Y. Moriura, et al. Phospholipase C-dependent $\mathrm{Ca} 2+$-sensing pathways leading to endocytosis and inhibition of the plasma membrane vacuolar $\mathrm{H}+-$ ATPase in osteoclasts. Am. J. Physiol. Cell Physiol. 299(3):C570-C578, 2010.

${ }^{16}$ Sanuki, R., C. Shionome, et al. Compressive force induces osteoclast differentiation via prostaglandin $\mathrm{E}(2)$ production in MC3T3-E1 cells. Connect. Tissue Res. 51(2):150-158, 2010.

${ }^{17}$ Tsuzuki, T., K. Okabe, et al. Osmotic membrane stretch increases cytosolic $\mathrm{Ca}(2+)$ and inhibits bone resorption activity in rat osteoclasts. Jpn. J. Physiol. 50(1):67-76, 2000.

${ }^{18}$ Wiebe, S. H., S. M. Sims, et al. Calcium signalling via multiple P2 purinoceptor subtypes in rat osteoclasts. Cell. Physiol. Biochem. 9(6):323-337, 1999.

${ }^{19}$ Xia, S. L., and J. Ferrier. Calcium signal induced by mechanical perturbation of osteoclasts. J. Cell. Physiol. 163(3):493-501, 1995.

${ }^{20} \mathrm{Xia}$, S. L., and J. Ferrier. Localized calcium signaling in multinucleated osteoclasts. J. Cell. Physiol. 167(1):148-155, 1996. 